\title{
Renale Ausscheidungsbedingungen von Cadmium beim normotensiven und hypertensiven Menschen
}

\author{
Untersucbungen ïber den Stoffwechsel von Spurenelementen, V. Mitteilung \\ Von D. P. Mertz, R. Koschnick und G. WilK \\ Aus der Medizinischen Poliklinik der Universität Freiburg i. Br. \\ und dem Chemischen Untersucbungsamt der Stadt Stuttgart
}

(Eingegangen am 21. Juni 1971)

An normotensiven und hypertensiven erwachsenen Versuchspersonen beiderlei Geschlechts mit normaler oder unterschiedlich eingeschränkter Nierenfunktion werden die renalen Ausscheidungsbedingungen von Cadmium bei unterschiedlichem Harnminutenvolumen untersucht. Die Bestimmung von Cd erfolgt mittels Emissionsspektralanalyse. Dabei wird folgendes festgestellt:

1. Die Ausscheidung von Cd beträgt bei nierensuffizienten Personen während cines mittleren Harnflusses von $4,71 \pm 2,50 \mathrm{ml} / \mathrm{Min}$. im Mittel 0,634 $\pm 0,521 \mu \mathrm{g} / \mathrm{Min}$., bezogen auf $1,73 \mathrm{~m}^{2}$ Körperoberfläche.

2. Sie steigt mit zunehmendem Harnminutenvolumen hochsignifikant an.

3. Bis zu einer Herabsetzung der Clearancewerte von Inulin auf weniger als $40 \mathrm{ml} / \mathrm{Min}$. und von PAH auf weniger als $200 \mathrm{ml} / \mathrm{Min}$. und $1,73 \mathrm{~m}^{2}$ Körperoberfläche wird $\mathrm{Cd}$ weitgehend unabhängig vom Verhalten der Nierenhämodynamik ausgeschieden.

4. Bei arterieller Hypertension besteht eine Tendenz zur verstärkten Ausscheidung von Cd. Keine Korrelation läßt sich zwischen den Serumkonzentrationen und Clearancewerten von $\mathrm{Cd}$ einerseits und der aktuellen Höhe des arteriellen Blutdruckes andererseits nachweisen.

\section{The renal excretion of cadmium in normotensive and bypertensive bumans}

The renal excretion of cadmium was investigated for different urine minute volumes in normotensive and hypertensive adult subjects of both sexes with normal or decreased kidney function. Cd was determined by emission spectral analysis. The following was established: 1. In persons with kidney sufficiency and with an urine flow of $4.71 \pm 2.50 \mathrm{ml} / \mathrm{min}$, the average excretion of $\mathrm{Cd}$ was $0.634 \pm 0.521 \mu \mathrm{g} / \mathrm{min}$ for $1.73 \mathrm{~m}^{2}$ of body surface. 2. This increases very significantly with an increasing rate of urine flow. 3. Down to a clearance value for inulin of less than $40 \mathrm{ml} / \mathrm{min}$ and for PAH of less than $200 \mathrm{ml} / \mathrm{min}$ and $1.73 \mathrm{~m}^{2}$ body surface area, the Cd excretion is largely independent of the kidney haemodynamics. 4. In arterial hypertension there is a tendency for an increased excretion of Cd. No correlation could be established between the serum concentration and clearance values of $\mathrm{Cd}$ on the one hand and the actual arterial blood pressure on the other hand.

Cadmium kommt neben vielen anderen verwandten Spurenelementen, wie Molybdän, Silber, Zink, Zinn und Blei, regelmäßig im Erwachsenen-Organismus vor und erscheint normalerweise im menschlichen Harn $(1,2)$. Eine biologische Funktion von $\mathrm{Cd}$ ist nicht bekannt. Beim Neugeborenen kann $\mathrm{Cd}$ nicht nachgewiesen werden (3). Mit zunehmendem Lebensalter reichert sich das Metall im menschlichen Organismus ständig an (4), weil die tägliche Zufuhr die Ausscheidung um etwa $3 \mu \mathrm{g}$ überschreitet (4). Nach dem dritten Lebensjahrzehnt bleibt die Cd-Konzentration im menschlichen Gewebe gleich, sie steigt dann in der 5. Dekade nochmals an $(4,5)$. Von der Akkumulation des Cd ist besonders die menschliche Niere betroffen (6). Im Mittel beträgt der Gehalt der Niere an Cd $40 \mathrm{mg} / \mathrm{kg}$ Feuchtgewicht (7). Radioaktives $\mathrm{Cd}$ wird hauptsächlich in Nierenrinde und Leber deponiert (8). Im Blut reichert sich radioaktives $\mathrm{Cd} \mathrm{zu} 90 \%$ in den Exythrocyten an (9). Ein erwachsener US-Amerikaner hat etwa $30 \mathrm{mg} \mathrm{Cd}$ in seinen Geweben abgelagert, davon allein 4,1 $\mathrm{mg}$ in der Leber (10).

Cd wird mit der Nahrung aufgenommen. Sehr stark cadmiumhaltig sind Schellfisch und Getreide (11). Weitere Quellen der Cd-Aufnahme sind Luftverschmutzung in industrialisierten Zonen und vermutlich
Zigarettenrauchen (12). Dieses Spurenelement ist eine starke Hemmsubstanz für Sulfhydryl-Enzyme (13), und verschiedene Vergiftungssymptome stehen mit der Theorie in Einklang, daß seine toxischen Effekte auf dieser Eigenschaft beruhen. In den Mitochondrien soll Cd eine Entkoppelung der oxydativen Phosphorylierung bewirken (14). In Nierenrindenschnitten von Ratten hemmt $\mathrm{Cd}$ ebenso wie verschiedene andere Metallionen ( $\mathrm{Zn}, \mathrm{Cu}$ ) die Gluconeogenese stark (15). Chronische Cd-Vergiftung durch Inhalation (17) kann beim Menschen und tierexperimentell eine chronische Bronchitis und Lungenemphysem hervorrufen. Etwa $70 \%$ des Cd-Gehaltes von Zigarettentabak gehen in den Rauch ein (17). Im Mittel enthalten 20 Zigaretten $20-30 \mu \mathrm{g} \mathrm{Cd}$, wovon $16 \%$ in der Asche und $15 \%$ in den Filtern, der Rest im Rauch erscheinen. Selbst wenn nur $10-20 \%$ der im Zigarettenrauch enthaltenen CdMenge inhaliert und absorbiert würden, hätte bei einer Person, die täglich mehr als 20 Zigaretten raucht, nach einigen Jahren eine wesentliche Anreicherung dieses Elementes im Organismus stattgefunden. Bei chronischer Intoxikation findet sich Cd in Lungen, Leber, Herz, Schilddrüse und Niere (18). Durch diätetischen Zusatz von Ascorbinsäure kann die Cd-Toxizität verringert werden (19). 
Anhand tierexperimenteller Untersuchungen wurde auf eine Beziehung zwischen Cd-Gehalt des Organismus und Bluthochdruck hingewiesen (20). Hypertensive Patienten scheiden $40 \mathrm{mal}$ soviel $\mathrm{Cd}$ aus wie normotensive Kontrollpersonen (21). Im 24-Stdn-Urin wurde bei einem durchschnittlichen Volumen von $1410 \mathrm{ml}$ eine mittlere Cd-Ausscheidung von $12,7 \pm 1,2$ (Spanne: $7,0-22,0) \mu \mathrm{g} / \mathrm{l}$ ohne signifikante Unterschiede zwischen Männern und Frauen, zwischen Schwarzen und Weißen gefunden (22). Änderung des pH-Wertes oder der Konzentration des ausgeschiedenen Harnes hatte keine erkennbare Wirkung auf die Ausscheidung. dieses Metalls im 24-Stdn-Urin, die für gewöhnlich nicht mehr als um $30 \%$ vom Mittelwert bei ein und derselben Versuchsperson schwankte. Nach anderen Angaben beträgt die durchschnittliche Cd-Ausscheidung im Urin 1,15 (Extremwerte: 0,5-10,8) $\mu \mathrm{g} / 1$ (23). Hauptausscheidungsorgan von $C d$ ist jedoch der Darm (24). Im menschlichen Serum bestimmten wir eine mittlere Cd-Konzentration von 258 (Extremwerte: 18 bis 1050) $\mu \mathrm{g} / 1$ (25).

Hinweise auf das Verhalten der renalen Eliminierung von $C d$ in Abhängigkeit vom Harnfluß oder von nierenhämodynamischen Meßgrößen konnten wir in der Literatur nicht finden. Vor allem interessierte uns in diesem Zusammenhang die Frage nach der $\mathrm{Ab}$ hängigkeit der renal ausgeschiedenen Cd-Menge von der Höhe des arteriellen Blutdruckes. Wir berichten hier über das Ergebnis diesbezüglicher eigener Untersuchungen.

\section{Methodik}

Untersucbungsgut

Die Untersuchungen fanden an insgesamt 29 Patienten, und zwar an 19 Männern und 10 Frauen, im Alter zwischen 19 und 61 Jahren (vgl. Tab. 1) statt. Es handelte sich dabei um 6 gesunde. Personen, um 10 Patienten mit chronisch-rezidivierender Pyelonephritis, von denen 8 normotensiv und 2 hypertensiv waten, um 9 Patienten mit chronischer Glomerulonephritis, wovon 3 normotensiv und 6 hypertensiv waren, um 2 Patienten mit essentieller Hypertension und um je einen hypertensiveh Patienten mit Nierenarterienstenose oder akuter Glomerulonephritis.

\section{Versuchsverfabren}

Die Versuche fanden unter denselben Bedingungen und unter Anwendung derselben analytischen Methoden statt, wie die vorausgegangenen Studien über die renale Ausscheidung von Eisen (26), Kobalt (27) und Nickel (28). Serienmäßig wurden bei jeder Versuchsperson im Nüchternzustand die Clearance- und Ausscheidungs̄werte von Inulin, $p$-Aminohippursäure $(\mathrm{PAH})$ und von Cadmium periodenweise bestimmt. Der Urin wurde durch Einführen eines Einmal-Plastik-Katheters in die Blase gewonnen und in spurenelementfreien Plastikgefäßen aufgefangen. Inulin bestimmten wir nach ROE und Mitarbeiter (29), PAH nach der von CzoK und Mitarbeitern (30) angegebenen Methode, Cadmium nach dem von uns früher mitgeteilten emissionsspektrographischen Verfahren (31).

\section{Versucbe bei unterscbiedlichem Harnfluß}

Alle Versuchspersonen tranken nach 12 stündigem Fasten 90 Min. vor Versuchsbeginn morgens innerhalb von 15-30 Min. 6-15 ml Flüssigkeit/kg Körpergewicht. Um die in der ersten Versuchsstunde auftretende Grunddiurese möglichst konstant $\mathrm{zu}$ halten, ließen wir im Bedarfsfalle stündlich 1-5 ml Flüssigkeit $/ \mathrm{kg}$ Körpergewicht nachtrinken. Die Cleatance-Perioden dauerten stets 30 Min.

Tab. 1

Verhalten der Ausscheidung und der Clearance von Cadmium bei unterschiedlichem Harnfluß $C_{\text {Inulin, }} \mathrm{PAH}_{\mathrm{A}} \mathrm{Cd}=$ Clearance von Inulin, $p$-Aminohippursäure bzw. Cadmium

\begin{tabular}{|c|c|c|c|c|c|c|c|c|c|}
\hline Proband & $\begin{array}{c}\text { Harn- } \\
\text { minutenvolumen } \\
(\text { ml/Min.) }\end{array}$ & $\begin{array}{c}\text { CInulin } \\
\text { (ml/Min.) }\end{array}$ & $\begin{array}{c}\mathrm{C}_{\text {PAH }} \\
\text { (ml/Min.) }\end{array}$ & $\begin{array}{l}\text { Cd Serum } \\
(\mu \mathrm{g} / \mathrm{l})\end{array}$ & $\begin{array}{l}\text { Cd Harn } \\
(\mu \mathrm{g} / \text { Min. })\end{array}$ & $\begin{array}{c}\mathrm{C}_{\mathrm{Cd}} \\
(\mathrm{ml} / \mathrm{Min} .)\end{array}$ & Klinische Diagnose & Alter & Geschlecht \\
\hline 1 & 2,44 & 113 & 369 & 270 & 0,678 & 2,49 & \multirow{11}{*}{$\begin{array}{l}\text { Chron. Glomerulonephritis, } \\
\text { Hypertension } \\
\text { Akute Glomerulonephritis, } \\
\text { Hypertension } \\
\text { Normalperson } \\
\text { Chron. Glomerulonephritis, } \\
\text { Hypertension } \\
\text { Chron. Glomerulonephritis, } \\
\text { Hypertension } \\
\text { Normalperson } \\
\text { Chron. Pyelonephritis } \\
\text { Chron. Pyelonephritis } \\
\text { Chron. Pyelonephritis } \\
\text { Nierenarterienstenose, } \\
\text { Hypertension } \\
\text { Chron. Pyelonephritis, } \\
\text { Hypertension } \\
\text { Normalperson } \\
\text { Essent. Hypertension } \\
\text { Chron. Pyelonephritis } \\
\text { Normalperson } \\
\text { Chron. Glomerulonephritis, } \\
\text { Hypertension } \\
\text { Essent. Hypertension } \\
\text { Chron. Glomerulonephritis } \\
\text { Chron. Pyelonephritis } \\
\text { Normalperson } \\
\text { Chron. Glomerulonephritis, } \\
\text { Hypertension } \\
\text { Normalperson } \\
\text { Chron. Glomerulonephritis } \\
\text { Chron. Pyelonephritis } \\
\text { Chron. Glomerulonephritis, } \\
\text { Hypertension } \\
\text { Chron. Pyelonephritis } \\
\text { Chron. Pyelonephritis, } \\
\text { Hypertension } \\
\text { Chron. Pyelonephritis } \\
\text { Chron. Glomerulonephritis }\end{array}$} & 43 & $\sigma^{\prime \prime}$ \\
\hline 2 & 6,09 & 69 & 281 & 800 & 0,755 & 0,94 & & 37 & $\sigma^{n}$ \\
\hline $\begin{array}{l}3 \\
4\end{array}$ & $\begin{array}{l}5,39 \\
5,28\end{array}$ & $\begin{array}{r}154 \\
81\end{array}$ & $\begin{array}{l}860 \\
256\end{array}$ & $\begin{array}{l}670 \\
137\end{array}$ & $\begin{array}{l}0,945 \\
0,671\end{array}$ & $\begin{array}{l}1,41 \\
4,88\end{array}$ & & $\begin{array}{l}34 \\
22\end{array}$ & $\stackrel{q}{q}$ \\
\hline 5 & 4,09 & 56 & 291 & 137 & 0,675 & 4,93 & & 45 & $\sigma^{\prime \prime}$ \\
\hline $\begin{array}{r}6 \\
7 \\
8 \\
9 \\
10\end{array}$ & $\begin{array}{l}8,63 \\
2,28 \\
4,16 \\
4,07 \\
2,34\end{array}$ & $\begin{array}{r}142 \\
88 \\
97 \\
68 \\
66\end{array}$ & $\begin{array}{l}537 \\
523 \\
455 \\
302 \\
125\end{array}$ & $\begin{array}{l}210 \\
520 \\
650 \\
350 \\
180\end{array}$ & $\begin{array}{l}0,028 \\
0,030 \\
0,045 \\
0,048 \\
0,583\end{array}$ & $\begin{array}{l}0,14 \\
0,06 \\
0,07 \\
0,14 \\
3,24\end{array}$ & & $\begin{array}{l}23 \\
28 \\
21 \\
26 \\
39\end{array}$ & $\begin{array}{l}o^{\prime} \\
0^{\prime} \\
0^{\prime \prime} \\
0^{\prime \prime} \\
\sigma^{\prime \prime}\end{array}$ \\
\hline 11 & 12,30 & 68 & 456 & 380 & 1,850 & 4,87 & & 28 & 우요 \\
\hline $\begin{array}{l}12 \\
13 \\
14 \\
15 \\
16\end{array}$ & $\begin{array}{l}6,12 \\
6,64 \\
1,62 \\
7,78 \\
2,46\end{array}$ & $\begin{array}{r}125 \\
101 \\
55 \\
130 \\
38\end{array}$ & $\begin{array}{l}520 \\
435 \\
323 \\
735 \\
225\end{array}$ & $\begin{array}{l}250 \\
180 \\
180 \\
250 \\
370\end{array}$ & $\begin{array}{l}1,360 \\
0,877 \\
0,458 \\
1,587 \\
0,891\end{array}$ & $\begin{array}{l}5,44 \\
4,87 \\
2,54 \\
6,35 \\
2,41\end{array}$ & & $\begin{array}{l}37 \\
39 \\
46 \\
26 \\
60\end{array}$ & $\begin{array}{l}0^{\prime \prime} \\
0^{\prime \prime} \\
o^{\prime \prime} \\
0 \\
0^{\prime}\end{array}$ \\
\hline $\begin{array}{l}17 \\
18 \\
19 \\
20 \\
21\end{array}$ & $\begin{array}{l}2,68 \\
2,26 \\
3,33 \\
6,09 \\
4,00\end{array}$ & $\begin{array}{r}85 \\
76 \\
85 \\
127 \\
38\end{array}$ & $\begin{array}{l}417 \\
563 \\
441 \\
679 \\
198\end{array}$ & $\begin{array}{r}220 \\
160 \\
27 \\
150 \\
20\end{array}$ & $\begin{array}{l}0,434 \\
0,355 \\
0,066 \\
1,050 \\
1,720\end{array}$ & $\begin{array}{r}1,97 \\
2,22 \\
2,44 \\
7,00 \\
86,00\end{array}$ & & $\begin{array}{l}49 \\
34 \\
21 \\
32 \\
61\end{array}$ & 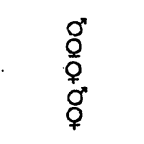 \\
\hline $\begin{array}{l}22 \\
23 \\
24 \\
25\end{array}$ & $\begin{array}{l}3,33 \\
2,91 \\
2,67 \\
7,85\end{array}$ & $\begin{array}{r}118 \\
73 \\
85 \\
78\end{array}$ & $\begin{array}{l}626 \\
538 \\
374 \\
606\end{array}$ & $\begin{array}{l}56 \\
18 \\
40 \\
40\end{array}$ & $\begin{array}{l}0,292 \\
0,271 \\
0,027 \\
0,785\end{array}$ & $\begin{array}{r}5,22 \\
15,10 \\
0,67 \\
19,60\end{array}$ & & $\begin{array}{l}49 \\
19 \\
30 \\
45\end{array}$ & $\begin{array}{l}0^{\prime \prime} \\
0^{\prime \prime} \\
0^{\prime \prime} \\
0\end{array}$ \\
\hline $\begin{array}{l}26 \\
27\end{array}$ & $\begin{array}{l}1,51 \\
6,74\end{array}$ & $\begin{array}{l}64 \\
77\end{array}$ & $\begin{array}{l}433 \\
339\end{array}$ & $\begin{array}{l}80 \\
50\end{array}$ & $\begin{array}{l}0,175 \\
1,040\end{array}$ & $\begin{array}{r}2,19 \\
20,80\end{array}$ & & $\begin{array}{l}51 \\
58\end{array}$ & $\begin{array}{l}0^{\prime} \\
0^{\prime \prime}\end{array}$ \\
\hline $\begin{array}{l}28 \\
29 \\
\end{array}$ & $\begin{array}{l}6,78 \\
4,71 \\
\end{array}$ & $\begin{array}{r}130 \\
39 \\
\end{array}$ & $\begin{array}{l}452 \\
138 \\
\end{array}$ & $\begin{array}{r}170 \\
45 \\
\end{array}$ & $\begin{array}{r}0,336 \\
-\quad 0,367 \\
\end{array}$ & $\begin{array}{l}1,98 \\
8,16 \\
\end{array}$ & & $\begin{array}{l}55 \\
40 \\
\end{array}$ & 옹 \\
\hline $\begin{array}{l}\bar{x} \\
\pm s\end{array}$ & $\begin{array}{l}4,71 \\
2,50\end{array}$ & 87 & 431 & 228 & $\begin{array}{l}0,634 \\
0,521\end{array}$ & 7,52 & & & $\cdot$ \\
\hline
\end{tabular}


Alle Probanden erhielten 3-4 Tage vor der Untersuchung eine standardisiertc Diät. Die tägliche Zufuhr von Natrium belief sich auf $40-70 \mathrm{mMol}$, diejenige von hochwertigem Protein auf $0,5-1,0 \mathrm{~g} / \mathrm{kg}$ Körpergewicht. Die Kost war vitaminreich und kalorisch ausreichend. Die Kaliumzufuhr wurde nicht beschränkt. Clearance-Werte und Ausscheidungsraten für $\mathrm{Cd}$ sind auf $1,73 \mathrm{~m}^{2}$ Körperoberfläche bezogen. - Die statistische Auswertung der Meßreihen erfolgte nach der $t$-Verteilung (STUDENT).

\section{Ergebnisse}

Die renale Ausscheidung von Cd zeigt nach Abbildung 1 einen signifikanten Anstieg mit zunehmendem Harnzeitvolumen. Bei einem mittleren Harnfluß von $4,71 \pm$ $2,50 \mathrm{ml} / \mathrm{Min}$. ergab sich für die Cd-Ausscheidung ein Mittelwert von $0,643 \pm 0,521 \mu \mathrm{g} /$ Min., bezogen auf $1,73 \mathrm{~m}^{2}$ Körperoberfläche. Wie Tabelle 1 ausweist, lag die Cd-Ausscheidung bei 7 der 12 hypertensiven Probanden über $0,7 \mu \mathrm{g} / \mathrm{Min}$., bei den restlichen 5 zwischen 0,7 und $0,4 \mu \mathrm{g} / \mathrm{Min}$. Dagegen eliminierten nur 4 der 17 normotensiven Personen mehr als $0,7 \mu \mathrm{g}$ $\mathrm{Cd} / \mathrm{Min}$. Bei einer normotensiven Versuchsperson bewegte sich die Cd-Ausscheidung zwischen 0,4 und $0,7 \mu \mathrm{g} /$ Min. Bei den restlichen 12 Patienten mit normalem Blutdruck betrug die Exkretion von Cd weniger als $0,4 \mu \mathrm{g} / \mathrm{Min}$., davon in 6 Fällen weniger als $0,1 \mu \mathrm{g} /$ Min.

Aus Abbildung 2 geht hervor, daß die renale CadmiumAusscheidung bei nierensuffizienten Personen weitgehend unabhängig vom Verhalten der nierenhämodynamischen Meßgrößen ist. Diese Feststellung trifft für eine Einschränkung der Inulin-Clearance bis auf Werte von weniger als $40 \mathrm{ml} / \mathrm{Min}$. und eine Herabsetzung der PAH-Clearance auf Werte von weniger als $200 \mathrm{ml} / \mathrm{Min}$. zu. Infolgedessen kann die große Schwankungsbreite der Cadmiumausscheidung nicht auf den unterschiedlichen Funktionszustand der Nieren zurückgeführt werden. Sehr wahrscheinlich stellt der Umstand, daß ein großer Teil unserer Versuchspersonen hypertensiv war, eine brauchbare Erklärung dar. So lagen denn auch die Wẹrte für die Cadmiumkonzentration im Urin um den Faktor 10 höher, als in den eingangs zitierten Literaturstellen angegeben ist. Keines-

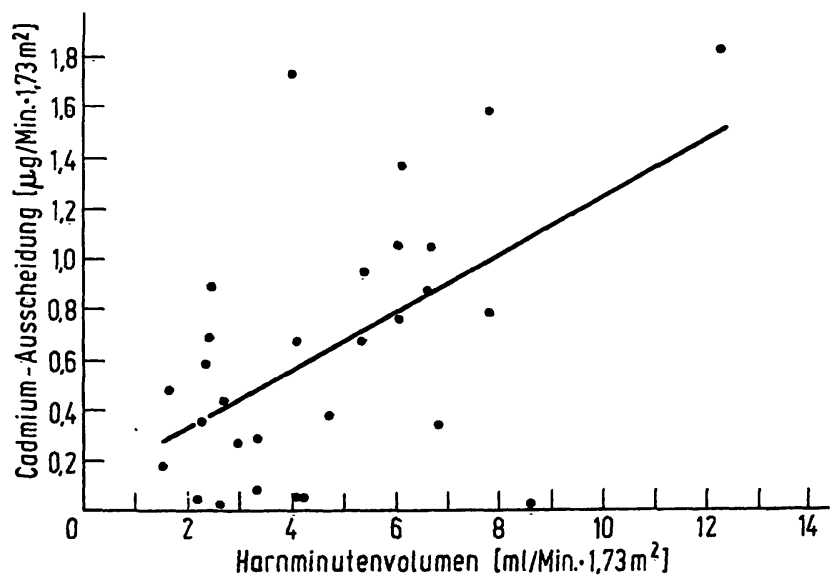

Abb. 1

Die Regressionsgerade zwischen Harnminutenvolumen und renale Cadmiumausscheidung bei indifferentem Harnfluß. $x=\bar{x} / \bar{y}$; $\mathrm{y}=0,114 \mathrm{x}+0,097 ; \mathrm{r}=0,55 ; \mathrm{p}<0,001(\mathrm{n}=29)$ wegs läßt sich nach unseren Befunden eine Parallelität der Serumkonzentration von $\mathrm{Cd}$ mit dem korrespondierenden arteriellen Blutdruck nachweisen (vgl. Tab. 1). Der von uns früher für die Serumkonzentration von Cd festgestellte Mittelwert von $258 \mu \mathrm{g} / 1$ (31), wurde von 4 der 12 hypertensiven und $6 \operatorname{der} 17$ normotensiven Versuchspersonen erreicht oder überboten. Bei den übrigen Probanden lagen die Werte zum 'Teil exheblich darunter. Ebensowenig lassen sich besonders hohe Werte für die Clearance von $\mathrm{Cd}$ einer Hypertension zuordnen. Eine Cd-Clearance von über $4 \mathrm{ml} / \mathrm{Min}$. wurde von 7 hypertensiven und 6 normotensiven Versuchspersonen erreicht.

\section{Diskussion}

Nach unseren Ergebnissen erweist sich die renale Ausscheidung von $\mathrm{Cd}$ bei nierensuffizienten Personen als deutlich abhängig vom Harnzeitvolumen, als weniger deutlich abhängig von der Höhe des arteriellen Blutdruckes und als unabhängig von nierenhämodynamischen Meßgrößen. Keine Korrelation scheint zwischen den Serumkonzentrationen und Clearancewerten von $\mathrm{Cd}$ einerseits und der aktuellen Höhe des arteriellen Blutdruckes andererseits zu bestehen. Die Unabhängigkeit der renal ausgeschiedenen Beträge an $\mathrm{Cd}$ von den Clearancewerten ergibt sich aus der individuell unter-

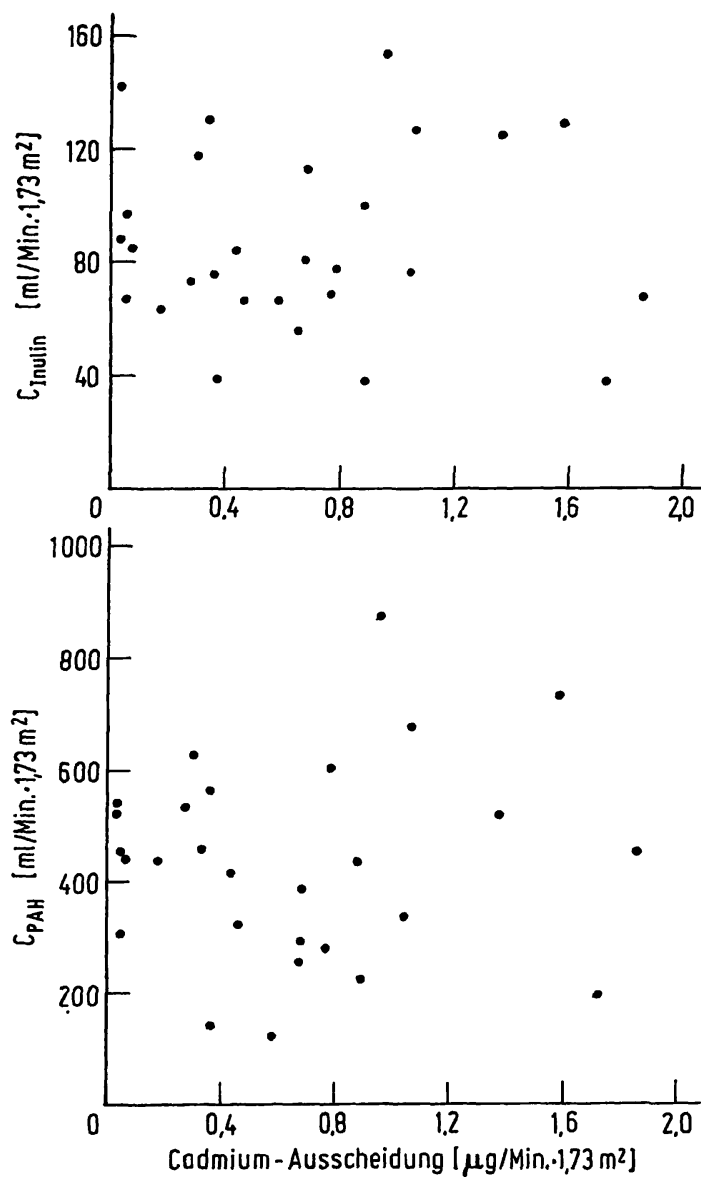

Abb. 2

Verhalten der renalen Ausschieidung von Cadmium im Vergleich zu den korrespondierenden Clearancewerten von Inulin $\left(C_{\text {Inulln }}\right)$ und PAH ( $\left.C_{1, A F}\right)$ bei indifferentem Harnfluß 
schiedlich eingeschränkten Nierenfunktion. Indessen bleibt jede Beziehung zwischen der tierexperimentell nachweisbaren akuten Vasoaktivität von $\mathrm{Cd}$ und der menschlichen Hypertension trotz einer erheblichen Anreicherung von $\mathrm{Cd}$ in menschlichen Nieren (32) und $\mathrm{dem}$ möglichen Vorhandensein ungewöhnlich hoher Cd-Beträge in Nieren von Hochdruckpatienten (33) problematisch. Übrigens konnte kürzlich eine Abhängigkeit des systolischen bzw. des diastolischen Blutdruckes von der Höhe der Cd-Ausscheidung nach Ausschaltung des Alterseinflusses statistisch nicht gesichert werden (34). Wenn Cd oder andere Spurenelemente mit Hypertension und Atherosklerose in Zusammenhang gebracht werden, ist es in Anbetracht der weitgehend ungeklärten Bedeutung dieser Elemente für definierte Stoffwechselvorgänge ungewiß, ob eine Beziehung kausal ist oder nicht (35). Möglicherweise wird das mit der Nahrung zugeführte $\mathrm{Cd}$ bei Fehlen einer renalen Ischämie eine gewisse Zeit lang durch die
Niere und durch die Leber aus der Zirkulation abgefangen, bis das Konzentrationsverhältnis von $\mathrm{Cd} z \mathrm{u} \mathrm{Zn}$ in der Niere einen gewissen Wert erreicht hat. Jenseits dieses Sättigungspunktes dürfte dann überschüssiges $\mathrm{Cd}$ peripher auf die Blutgefäße einwirken und eine Vasokonstriktion erzeugen. Das in der Nierenrinde vorhandene $\mathrm{Cd}$ ist an ein Protein, das Metallothionein $(36,37)$, gebunden. Die Wirkung von $\mathrm{Cd}$ auf den proximalen Nierentubulus besteht' ${ }^{\prime}$ in einer Förderung der Reabsorption von Natrium mit einer vermehrten Wasserresorption $(38,39)$. Die Existenz von Metallothionein legt die Vermutung nahe, daß Cd eine physiologische Rolle bei der Reabsorption von Natrium im proximalen Tubulus der Niere spielt.

Inwieweit Rauchergewohnheiten $\mathrm{zu}$ den individuellen Besonderheiten und der großen Schwankungsbreite der Werte von Versuchsperson zu Versuchsperson beigetragen haben, läßt sich im einzelnen jetzt nicht mehr übersehen.

\section{Literatur}

1. Tipton, I. H., M. J. Cook, R. L. Steiner, J. M. Foland, K. K. McDaniel und S. D. Fentress, Oak Ridge National Laboratory Report, C. F. 57-2-4, February 28, 1957. - 2. PERRY, H. M., jr. und E. F. Perry, J. clin. Invest. 38, 1452 (1959). - 3. SCHroeder, H. A., Adv. internat. Med. 8, 259 (1956). - 4. Tipron, J. H., in: Metal Binding in Medicine, ed. by M. J. Seven and L. A. Johnson. Lippincott, Philadelphia (1960). - 5. Schroeder, H. A. und J. J. Balassa, J. chron. Dis. 14, 236 (1961). - Tipton, J. H., W. D. Foland, F. C. BoвB und W. C. MCCoRCKLE, Oak Ridge National Laboratory Report, C. F. 53-8-4, March 11, 1953. 7. Perry, H. M., jr., J. H. Tipton, H. A. Schroeder, L. R. Steiner und M. J. Cook, J. chron. Dis. 14, 259 (1961): - 8. GunN, S. A. und T. C. Gound, Proc. Soc. Exper. Biol. N. Y. 96, 820 (1957). - 9. Moeschrin, S., Klinik und Therapie der Vergiftungen, 4. Aufl., G. Thieme, Stuttgart (1964). - 10. Report of I. C. R. P. Committee II on Permissible Dose for Internal Radiation, Hlth Phys. 3, 1 (1960). - 11. Schroeder, H. A., J. J. Balassa und I. H. Tipton, J. chron. Dis. 15, 941 (1962). 12. Schroeder, H. A., J. J. Balassa und J. C. Hogencamp, J. chron. Dis. 14, 236 (1961). - 13. Forster, R. P. und J. V. TAGGART, J. Cellular Comp. Physiol. 36, 251 (1950). - 14. JACOBS, E. E., M. Jacob, D. R. Sanadr und L. B. Bradley, J. biol. Chemistry 223, 147 (1956). - 15. Rutman, J. Z., L. E. Meltzer, J. R. Kitchell, R. J. Rutman und P. George, Amer. J. Physiol. 208, 841 (1965). - 16. Lewis, G. P., H. LYLE und S. MILLER, Lancet 1969/II, 1330. - 17. NANDI, M., H. JiCK, D. SLone und S. Shapiro, Lancet 1969/II, 1329. - 18. Schroeder, H. A. und J. J. Balassa, J. chron. Dis. 14, 236 (1961). - 19. Fox, M. R. S. und B. E. FRY, jr., Science 169, 989 (1970). - 20. SCHROEDER, H. A., H. M. Perry, jr., E. G. Dennis und L. E. Mahoney, J. exper. Med. 102, 319 (1955). - 21. Perry, H. M., jr. und H. A. SChroeder, J. Laborat. Clin. Med., S. Louis 46, 936 (1955). 22. Perry, H. M., jr. und E. F. Perry, J. clin. Invest. 38,1452 (1959). - 23. Imbus, H. R., J. Cholak, L. H. Miller und T. Sterling, Arch. environm. Hlth. 6, 286 (1963). - 24. Walch, J. J. und G. E. Burch, J. Laborat. Clin. Med. 54, 59 (1959). 25. Mertz, D. P., G. Wrik und R. Koschnick, Verh. Dtsch. Ges. Inn. Med. 74, 600 (1968). - 26. Mertz, D. P., R. KosChNicK und G. Wrrk, diese Z. 7, 1 (1969). - 27. MERTZ, D. P., G. WrLK und R. Koscrinrck, diese Z. 8, 30 (1970). - 28. Mertz, D. P., R. Koschnsck und G. WIrk, diese Z. 8, 387 (1970). - 29. RoE, J. H., J. H. Epstern und N. P. Goldstenn, J. biol. Chemistry 178, 839 (1949). - 30. Czok, G., W. Kreienberg und D. P. Mertz, Klin. Wschr. 30, 227 (1952). - 31. Mertz, D. P., R. Koschinick, G. Wilk und K. Pfeilsticker, diese Z. 6,171 (1968). - 32. Perry, H. M., jr., I. H. Tipton, H. A. Schroeder, R. L. Steiner und M. J. Cook, J. chron. Dis. 14, 259 (1961). 33. Schroeder, H. A., J. chron. Dis. 18, 647 (1965). - 34. SzadKowskr, D., K.-H. Schaller und G. Lefinert, diese Z. 7, 551 (1969). - 35. Mertz, D. P., Elektrolytstoffwechsel und arterielle Hypertension. F. K. Schattauer, Stuttgart, New York (1971). - 36. KäGI, J. H. R. und B. L. VALLEE, J. biol. Chemistry 235, 3460 (1960). - 37. KäGr, J. H. R. und B. L. VALLEE, J. biol. Chemistry 236, 2435 (1961). - 38. VANDER, A. J., Amer. J. Physiol. 203, 1 (1962). - 39. Vander, A. J., Amer. J. Physiol. 203, 1005 (1962).
Prof. Dr. D. P. Mertz Med. Univ. Poliklinik 78 Freiburg i. Brsg. Hermann-Herder-Str. 6 